DOI : $10.22199 / \mathrm{S} 07160917.1982 .0001 .00002$

\title{
UNA HISTORIA DEL DEPARTAMENTO DE MATEMATICAS
}

OSCAR ROJO J. (Ph. D.)

Frente a la tarea de escribir algunas líneas relativas a una historia del Departamento de Matemáticas, he creído necesario retroceder en el tiempo y ubicarme en mis primeros años de estudiante de esta Universidad y, desde allí, volverá caminar hasta el momento actual. Claramente, se observan tres etapas en el desarrollo que ha tenido la disciplina matemática en nuestra Universidad. El análisis de cada una de ellas nos dará una exacta perspectiva para mirar el momento actual de esta disciplina en nuestra casa de estudios. Por otra parte, una historia del Departamento que no considere la evolución que ha tenido la Matemática entre nosotros, no pasaría más allá de una enumeración de hechos sin ninguna conexión entre ellos. 
lera. ETAPA: PRIMEROS AÑOS DE LA MATEMATICA EN LA UNIVERSIDAD DEL NORTE ( $\left.\begin{array}{ll}1957 & 1964\end{array}\right)$

La docencia en Matemáticas en la Universidad del Norte empieza con la creación de la Universidad. Inicialmente, con el Primer año de Ingeniería, cuyos alumnos continuaban después sus estudios en la Universidad Católica de Valparaíso y, desde el segundo año de existencia de la Universidad, con la Escuela de Pedagogía en Matemáticas y Física, hoy día llamada Carrera de Pedagogía en Matemáticas y Física. Enseñaron Matemática en esta primera etapa entre otros:

- Don Aníbal Gálvez Z., quien por casi 10 años fue el Director de la Escuela de Matemáticas y Física, y uno de los principales impulsores de la Creación del Departamento de Matemáticas.

- Don Juan Mandaleris K. (Q.E.P.D.). Ejerció desde la creación de la Universidad hasta 1964 en esta ciudad, trasladándose en 1965 a la ciudad de Arica, lugar en el cual empezaba a funcionar la ex sede Arica de nuestra Universidad.

- Sra. Minerva Chilovitis R., que trabajó en nuestra Universidad hasta fines de 1959.

- Don Osvaldo Garay 0., quien 1lega a muestra Universidad en 1959, como Director de la Escuela de Matemáticas y Física y posteriormente ocupa importantes cargos directivos llegando a ser Secretario General de la Universidad. Abandona nuestra Universidad en 1965, para hacerse cargo de la Superintendencia de Educación. 
- Don Ricardo López y Sra. Leontina Espinoza de López, matrimonio de matemáticos que ejerció desde 1959 a 1961 en nuestra casa de estudios.

- R.P. Germán Saá (Q.E.P.D.), persona muy querida por su notable calidad humana y uno de los primeros científicos de la Universidad. En Biblioteca se pueden encontrar textos de Matemática que él donó a la Universidad del Norte.

La docencia en esta primera etapa consiste en cursos de: Algebra Clásica, Cálculo Diferencial e Integral, Cálculo Avanzado, Análisis Vectorial y cursos de Geometría. Esta docencia estaba destinada a los alumnos de la Escuela de Matemáticas y Física y a las carreras de Ingeniería que en ese tiempo existían. Ella era ejercida por dos grupos de docentes sin ninguna conexión.

No se aprecia en este período una actividad de Investigación Matemática.

Entre las actividades de trascendencia realizadas en esta etapa, podemos mencionar un curso de Algebra Moderna, dictado en el Invierno de 1964 por el Doctor en Matemáticas, don Elemer Nemesszeghy, quien en ese entonces trabajaba en la Universidad Católica de Valparaíso y quién aceptó una invitación que le cursara la Escuela de Matemáticas y Física. Es el primer curso de Algebra Moderna que se dictan en la ciudad. Se comienza a hablar de Teoría de Conjuntos, Teoría de Grupos, Tweoría de Anillos, Teoría de Cuerpos, y de Algebra de Boole. 
2da. ETAPA: AÑOS PREVIOS A LA CREACION DEL

DEPARTAMENTO DE MATEMATICAS (1965-1968)

A partir de 1963, se produce la incorporación a la planta académica de la Universidad de algunos egresados de la Carrera de Matemáticas y Física. Entre ellos tenemos :

H. Raúl Muñoz E.
Orlayer Alcayaga M.
Carlos Mendizábal J.
Oscar Rojo J.
Néstor Aviles R.
Fredy Veas M.
David Lazo
Hugo Mendizábal J.
Rodolfo Baeza V.
Eduardo Muñoz M.
Héctor Rojo J.
Ljubomir Bacovi R.

H. Raúl Muñoz E.

Orlayer Alcayaga M.

Carlos Mendizábal J.

Oscar Rojo J.

Néstor Aviles R.

Fredy Veas M.

David Lazo

Hugo Mendizábal J.

Rodolfo Baeza V.

Héctor Rojo J.
$(1963)$

(1963)

(1964)

(1964)

(1964)

(1965)

(1965)

(1966)

$(1966)$

(1967)

(1967)

(1967)

Raúl Muñoz, Orlayer Alcayaga (hoy día, Doctor en Física), y Néstor Avilés orientaron su quehacer hacia la Física, disciplina en la cual han alcanzado un alto nivel de excelencia. Carlos Mendizábal, Fredy Veas M. y David Lazo ejercieron desde 1965 en la ex -sede Arica de la Universidad. Hugo Mendizábal trabajó un tiempo en esta ciudad,posteriormente en la ex sede Arica y hace pocos años alcanzó un Doctorado en Física. Los restantes, es decir, Oscar Rojo, Rodolfo Baeza, Eduardo Muñoz, Héctor Rojo y Ljubomir Bacovi orientaron su interés hacia la Matemática, integrándose el primero de éstos al cuerpo docente de la Escuela de Matemáticas y Física y los otros al grupo que atendía 
la docencia en Matemáticas de las carreas de Ingeniería, más precisamente a la unidad denominada PACITUN (Primer Año Común del Instituto Tecnológico de la Universidad de1 Norte). Se incorporan además a esta unidad, los profesores:

$$
\begin{array}{ll}
\text { Arnoldo Prado C. } & \text { (1966) y } \\
\text { Alfredo Vásquez S. (1967) }
\end{array}
$$

Ambos realizaron sus estudios en la Universidad Católica de Valparaíso; el Profesor Prado ejerció la Dirección del PACITUN desde 1967 hasta la creación del Departamento de Matemáticas.

Tenemos así dos grupos que ejercen la docencia en Matemáticas; por una parte, el grupo que atendía a la Escuela de Matemáticas y, por otra parte, el grupo que atendía las carreras tecnológicas. Estos dos grupos formarían el primer cuerpo docente del Departamento.

Se observa en esta segunda etapa, una modernización en los contenidos de ciertas asignaturas: se empieza a enseñar Teoría de Conjuntos, Estructuras Algebraicas (grupos, anillos y cuerpos), Algebra Lineal, y la Geometría es enseñada en forma axiomática. Contribuyó en parte a este logro la presencia, por un par de años, en la Escuela de Matemáticas y Física del Licenciado en Matemáticas Sr. Pierre Duval, de nacionalidad francesa.

Asiste a un Seminario de Perfeccionamiento en Matemáticas, realizado en la ciudad de Santiago y organizada por el Ministerio de Educación, el Profesor Oscar Rojo J., durante los meses de Junio y Julio de 1966. E1 Profe- 
sor Rodolfo Baeza asiste en 1967 a un Simposio de Matemáticas realizado en la ciudad de Buenos Aires. Sin embargo, el perfeccionamiento académico es todavía débil y no obedece a una planificación. Se aprovechan algunas visitas ocasionales de doctores en Matemáticas. Podemos mencionar al Doctor Herbert Clemens y al Doctor Malcom Tobey.

A nivel de Universidad se analizan ciertas propuestas de reformas estructurales. Entre ellas, se estudia la conveniencia de considerar al Departamento como unidad básica de la actividad académica. A nivel de los grupos que atendían la docencia en Matemáticas, se considera la idea de Departamento como algo necesario y conveniente para mejorar el nivel de la actividad matemática. Es así, como con fecha 24 de Octubre de 1968 los Profesores Arnoldo Prado y Oscar Rojo presentan un Proyecto de Departamento de Matemáticas a sis colegas. Por otra parte, el Profesor Aníbal Gálvez, presenta a las autoridades un Proyecto para la creación de un Departamento de Matemáticas. Finaliza así el año 1968 .

3era. ETAPA: CREACION Y DESARROLLO DEL DEPARTAMENTO DE MATEMATICAS (desde 1969).

Con fecha 8 de Marzo de 1968, y según Decreto 6/69, la Universidad es estructurada académicamente en Areas y cada área en Departamentos. Entre estos figura la creación del Departamento de Matemáticas. Se agrupan así en una misma unidad todos los académicos que ejercían docencia matemática. Estos eran:

Ljubomir Bacovi R. Rodolfo Baeza V. 

Aníbal Gálvez Z.
Eduardo Muñoz M.
Arnoldo Prado C.
Héctor Rojo J.
Oscar Rojo J.
Alfredo Vásquez S.

A estos nombres debemos agregar los profesores Eduardo Camino E., Mario del Campo y Eduardo Labarca, contratados inmediatamente después de la creación del Departamento. E1 mismo decreto de creación antes mencionado designó a los Directores de Departamento, siendo designado como Director de nuestro Departamento en carácter de interino, el Profesor Arnoldo Prado C.. Con fecha 1 de Julio de 1969 fue designado como Director Titular el Profesor Oscar Rojo J. Han ocupado la Dirección del Departamento de Matemáticas y en los períodos que en cada caso se indican, los profesores:

$\begin{array}{ll}\text { Arnoldo Prado C., } & (08.3 .69-01.7 .69) \\ \text { Oscar Rojo J. } & (01.7 .69-05.3 .70) \\ \text { Aníbal Gálvez Z. } & (05.3 .70-01.5 .70) \\ \text { Héctor Rojo J. } & (01.5 .70-16.3 .71) \\ \text { Alfredo Vásquez S. } & (16.3 .71-31.7 .71) \\ \text { Oscar Rojo J. } & (01.8 .72-27.6 .73) \\ \text { Rodolfo Baeza V. } & (27.6 .73-23.6 .75) \\ \text { Alfredo Vásquez S. } & (23.6 .75-01.3 .78) \\ \text { Oscar Rojo J. } & (01.3 .78-01.4 .79) \\ \text { Rodolfo Baeza V. } & (01.4 .79-09.6 .80) \\ \text { Oscar Rojo J. } & (09.6 .80-31.8 .81) \\ \text { Eduardo Muñoz M. } & (31.8 .81-\end{array}$

Se entrega al Departamento la responsabilidad 
de ejercer la docencia en todas las asignaturas matemáticas, como también la responsabilidad del cultivo de esta ciencia. Junto con el inicio de sus actividades, el Departamento de Matemáticas realiza un análisis profundo del estado de la disciplina matemática en la Universidad y del grado de preparación de los académicos integrantes del Departamento. Como resultado de este estudio, surgió la necesidad imperiosa de perfeccionamiento que permitiera, al menos, mejorar y afrontar con éxito la función docente universitaria en Matemáticas como elemento previo y necesario para el cultivo de la disciplina. Se formulan, junto con el transcurso de los años, varios planes de perfeccionamiento, todos coherentes entre sí. Destaca, en particular, el Plan de Perfeccionamiento presentado con fecha 15 de Junio de 1973, plan que mereció las felicitaciones del entonces denominado Comité Permanente de Perfeccionamiento Académico y que fue la base fundamental de planes posteriores. Debemos dejar constancia del apoyo decidido que siempre hemos recibido de las autoridades universitarias, para nuestros deseos de perfeccionamiento y el apoyo otorgado, los siguientes académicos han obtenido el o los grados académicos que en cada caso se indica:

- Alfredo Vásquez S., Máster en Estadística Matemáticas (1970), Programa CIENES, Santiago de Chile. Realiza estudios conducentes al doctorado en la Universidad de Delawere, Estados Unidos.

- Oscar Rojo J., Magister en Matemáticas, (1971) Universidad Técnica del Estado, Santiago de Chile. Doctor of Philosophy in Mathematics (1976), New Mexico State University. 
- Arnoldo Prado C., Magister en Estadística Matemática (1972), Universidad Católica de Valparaíso. Doctor en Ciencias Matemáticas (1982), Universidad Complutense de Madrid, España.

- Rodolfo Baeza V., Magister en Matemáticas (1972), Universidad Técnica del Estado, Santiago de Chile. Estudios de doctorado realizados en el Instituto de Matemáticas Pura y Aplicada, Brasil.

- Eduardo Muñoz M., Magister en Matemáticas (1973), Universidad Técnica del Estado, Santiago de Chile.

- Eduardo Camino E., Magister en Matemáticas (1973), Universidad Técnica del Estado, Santiago de Chile.

- Marcia Rojas S., Licenciado en Matemáticas (1978), Universidad del Norte.

- Raúl Zhigley C., Master en Estadística Matemática (1977), Programas CIENES, Antofagasta y Santiago de Chile.

- Ricardo Soto M., Magister en Matemáticas (1978), Universidad Técnica del Estado, Antofagasta y Santiago de Chile.

- Patricio Rojas M., Master en Estadística Matemática (1978), Programa CIENES, Antofagasta y Santiago de Chile.

Debemos también mencionar a María Leal R., quién con el apoyo de la Universidad realizó estudios de docto- 
rado en España.

Vemos así el efecto que han tenido, nuestros planes de perfeccionamiento, en la planta académica del Departamento. Es de justicia, además, decir alguna consecuencias del efecto multiplicador que este perfeccionamiento ha implicado. Esto se ha materializado en varios cursos de perfeccionamiento dictados a colegas del Departamento de Matemáticas, como también a colegas de otros Departamentos, que han permitido transmitir conocimientos que anteriormente eran adquiridos en otros lugares.

Esto también ha sido posible con la incorporación a nuestra planta académica de:

- Luis Valdivia R. (1974), Magister en Matemáticas, Universidad Técnica del Estado, Santiago.

- Washington Mancilla (1977) (Q.E.P.D.).

- María Soledad Romo (1978), Magister en Matemáticas, Universidad Técnica del Estado, Santiago y con estudios de doctorado en Francia.

A 10 anteriormente mencionado, debemos agregar que el nivel alcanzado por la actual planta académica del Departamento ha permitido elevar notablemente el nivel de la docencia universitaria en matemáticas y tener en ejecución tres programas de investigación. Estos logros han sido obtenidos gracias a la integración, en una misma unidad académica, de quienes ejercemos la función de matemáticos en la Universidad. E1 futuro nos ofrece interesantes desafíos. Tenemos por un lado nuevas exigencias 
docentes, la Universidad se desarrolla y surgen así por otra parte, nuevos requerimientos de Matemáticas. Tenemos, también, el desarrollo mismo de las Matemáticas, que nos obliga a un permanente estudio y la investigación matemática debes der una materia prioritaria. 